\title{
Controllability of nonlocal non-autonomous neutral differential systems including non-instantaneous impulsive effects in $\mathbb{R}^{n}$
}

\author{
Velusamy Kavitha, Mani Mallika Arjunan* and Dumitru Baleanu
}

\begin{abstract}
This manuscript involves a class of first-order controllability results for nonlocal non-autonomous neutral differential systems with non-instantaneous impulses in the space $\mathbb{R}^{n}$. Sufficient conditions guaranteeing the controllability of mild solutions are set up. Concept of evolution family and Rothe's fixed point theorem are employed to achieve the required results. A model is investigated to delineate the adequacy of the results.
\end{abstract}

\section{Introduction}

Some real-life procedures can be described by quick changes in their state. A portion of these progressions start incautiously and are not insignificant. For changes, for example, these, scientific models called non-instantaneous differential conditions are made. These models offer ascent to another, half and half dynamical system that can be utilized for various purposes. The investigation on non-abrupt impulses be one of present concern of several researchers $[1,2,8,13,4,6,9,16,14]$. Hernandez and O'Regan [8] and Pierri et al. [13], initially analyzed Cauchy problems for first order evolution equations with non-instantaneous impulses. Lately, Wang and Feckan [17] generalized

Key Words: Controllability, Nonlocal conditions, Non-instantaneous impulsive conditions, Non-autonomous differential systems, Mild solution, Fixed point.

2010 Mathematics Subject Classification: Primary 93B05; Secondary 34A37.

Received: 22.12 .2019

Accepted: 17.02 .2020 
CONTROLLABILITY OF NONLOCAL NON-AUTONOMOUS NEUTRAL DIFFERENTIAL SYSTEMS INCLUDING NON-INSTANTANEOUS IMPULSIVE EFFECTS IN $\mathbb{R}^{n}$

the results of [8] under different conditions. For further information, please reader to refer $[8,13,6,9]$.

The nonlocal issue was driven by physical issues. Moreover, it has been shown that nonlocal problems have a stronger impact on applications than the classic Cauchy problems. It is used, for instance, to depict mathematical models for the evolution of multiple phenomena such as nonlocal neutral networks, nonlocal pharmacokinetics, and non-local pollution (see $[5,7]$ ).

The idea of controllability indicates the capacity to move a framework around in its whole configuration space utilizing just certain allowable controls. The perfect mathematical definition shifts marginally inside the structure or the sort of models applied. Nowadays, there are numerous intriguing uses of control concept, for instance, reactor, biological networks, chemical technology and power system control, in general, concept of control theory used in different branches of science and technology etc $[15,3,12,10,11]$. In [10], author discussed the controllability of non-autonomous systems with the help of Rothe's fixed point theorem. Further, the same author [11] studied the controllability of semilinear impulsive differential systems in $\mathbb{R}^{n}$ under some suitable conditions and Rothe's fixed point theorem. Lately, Malik et al. [12] analyzed the controllability results for non-autonomous nonlinear differential systems with non-instantaneous impulses under suitable fixed point theorem.

As per the authors knowledge, there is no work yet reported on noninstantaneous impulsive neutral non-autonomous differential system with nonlocal conditions in $\mathbb{R}^{n}$. For this reason, to bridge this crevice, we deal with a nonlocal non-autonomous neutral differential systems including non-abrupt impulsive effects in $\mathbb{R}^{n}$.

Inspired by the above mentioned works $[17,12,11]$, in this manuscript, we discuss a non-autonomous nonlocal neutral differential system along nonabrupt impulsive effects of the form

$$
\begin{aligned}
\frac{d}{d t}[w(t)-\mathscr{H}(t, w(t))] & =A(t)[w(t)-\mathscr{H}(t, w(t))]+B(t) u(t)+\mathscr{F}(t, w(t)) \\
t \in \bigcup_{q=0}^{p}\left(s_{q}, t_{q+1}\right) & \\
w(t) & =\xi_{q}\left(t, w\left(t_{q}^{-}\right)\right), \quad t \in \bigcup_{q=1}^{p}\left(t_{q}, s_{q}\right] \\
w(0) & =w_{0}+r(w) \in \mathbb{R}^{n}
\end{aligned}
$$

where $w(t) \in \mathbb{R}^{n}, 0=s_{0}=t_{0}<t_{1}<s_{1}<t_{2}, \cdots, t_{p}<s_{p}<t_{p+1}=T<$ $\infty, u(t) \in \mathbb{R}^{m}$. From equation (1.2), we notice that $w \in C\left(\left(t_{q}, t_{q+1}\right], \mathbb{R}^{n}\right), q=$ $0,1, \ldots, p$ and we can find $w\left(t_{q}^{-}\right)$and $w\left(t_{q}^{+}\right), q=1,2, \ldots, p$ with $w\left(t_{q}^{-}\right)=$ 
CONTROLLABILITY OF NONLOCAL NON-AUTONOMOUS NEUTRAL DIFFERENTIAL SYSTEMS INCLUDING NON-INSTANTANEOUS IMPULSIVE

$w\left(t_{q}\right)$. The functions $\xi_{q}\left(t, w\left(t_{q}^{-}\right)\right)$constitute the non-instantaneous impulses during the interval $\left(t_{q}, s_{q}\right], q=1,2, \ldots, p$, thus impulses at $t_{q}$ have some duration, namely on intervals $\left(t_{q}, s_{q}\right]$. Moreover $A(t)$ and $B(t)$ defined as a matrices of $n \times n$ and $n \times m$, separately. The functions specified in (1.1)-(1.3) are defined further section appropriately.

The scheme about manuscript is as stated. Within the to begin with and second segments, we present the presentation, crucial definitions and groundwork which are utilized all through this manuscript. In section 3, authors demonstrate the controllability of the linear control framework comparing the issue (1.1)-(1.3) without impulses. Further, we built up the adequate setting for the controllability of nonlocal non-autonomous neutral differential systems with non-instantaneous impulses of the system (1.1)-(1.3) with the help of Rothe's fixed point theorem in section 4. In the last section, an example is given to show the application of the obtained results.

\section{Preliminary Results and Hypotheses}

In this section, we recall some basic definitions, notations and theorem which will be used throughout this manuscript.

Let $\mathscr{P} \mathscr{C}\left([0, T], \mathbb{R}^{n}\right)$ be the space of piecewise continuous functions. That is, $\mathscr{P} \mathscr{C}\left([0, T], \mathbb{R}^{n}\right)=\left\{w: \mathscr{J}=[0, T] \rightarrow \mathbb{R}^{n}: w \in C\left(\left(t_{j}, t_{j+1}\right], \mathbb{R}^{n}\right), j=\right.$ $0,1,2, \ldots, p$, and we can find $w\left(t_{j}^{-}\right)$and $w\left(t_{j}^{+}\right), j=1,2, \ldots, p$ with $w\left(t_{j}^{-}\right)=$ $\left.w\left(t_{j}\right)\right\}$, supplied with the supremum criterion

$$
\|w\|_{0}=\sup _{t \in \mathscr{J}}\|w(t)\|_{\mathbb{R}^{n}} .
$$

Presently, we treat $\mathscr{P} \mathscr{C}(\mathscr{J}) \times \mathscr{C}(\mathscr{J})=\mathscr{P} \mathscr{C}\left(\mathscr{J}, \mathbb{R}^{n}\right) \times \mathscr{C}\left(\mathscr{J}, \mathbb{R}^{m}\right)$ is a Banach space supplied with the subsequent criterion

$$
\|(w, u)\|_{0}=\|x\|_{0}+\|u\|_{0} .
$$

In $\mathbb{R}^{n} \times \mathbb{R}^{m}$, we treat the subsequent criterion

$$
\|(w, u)\|_{1}=\|w\|_{\mathbb{R}^{n}}+\|u\|_{\mathbb{R}^{m}}=\|w\|+\|u\|, \quad \forall(t, w) \in \mathbb{R}^{n} \times \mathbb{R}^{m} .
$$

Each $w \in \mathscr{P} \mathscr{C}(\mathscr{J})$, we characterize the subsequent criterion

$$
\|\mathscr{H}(\cdot, w)\|_{0}=\sup _{t \in \mathscr{J}}\|\mathscr{H}(t, w(t))\|_{\mathbb{R}^{n}}
$$

and

$$
\|\mathscr{F}(\cdot, w)\|_{0}=\sup _{t \in \mathscr{J}}\|\mathscr{F}(t, w(t))\|_{\mathbb{R}^{n}} .
$$


CONTROLLABILITY OF NONLOCAL NON-AUTONOMOUS NEUTRAL DIFFERENTIAL SYSTEMS INCLUDING NON-INSTANTANEOUS IMPULSIVE EFFECTS IN $\mathbb{R}^{n}$

Definition 2.1. The problem (1.1)-(1.3) is said to be controllable on $\mathscr{J}$ if for every $w(0), w_{1} \in \mathbb{R}^{n}$, we can find $u \in \mathscr{L}^{2}\left(\mathscr{J}, \mathbb{R}^{n}\right)$ in a way that the solution $w(t)$ of the given problem (1.1)-(1.3) analogous to $u$ fulfills

$$
w(0)=w_{0}+r(w) \quad \text { and } \quad w(T)=w_{1} .
$$

To establish the primary result of the problem (1.1)-(1.3), now, we list the subsequent assumptions:

(H1) The function $\mathscr{F}: \mathscr{J}_{1} \times \mathbb{R}^{n} \rightarrow \mathbb{R}^{n}, \mathscr{J}_{1}=\cup_{q=0}^{p}\left[s_{q}, t_{q+1}\right]$ is continuous, in a way that

$$
\|\mathscr{F}(t, w)\|_{\mathbb{R}^{n}} \leq \gamma_{0}\|w\|_{\mathbb{R}^{n}}^{\beta_{0}}+\delta_{0}, t \in \mathscr{J}_{1}, w \in \mathbb{R}^{n}
$$

with $0<\beta_{0} \leq 1$

(H2) The function $\mathscr{H}: \mathscr{J}_{1} \times \mathbb{R}^{n} \rightarrow \mathbb{R}^{n}$ is continuous and we can find a positive constant $\mathscr{L}_{\mathscr{H}}$ in a way that

$$
\left\|\mathscr{H}\left(t, w_{1}\right)-\mathscr{H}\left(t, w_{2}\right)\right\|_{\mathbb{R}^{n}} \leq \mathscr{L}_{\mathscr{H}}\left\|w_{1}-w_{2}\right\|_{\mathbb{R}^{n}}
$$

for every $w_{1}, w_{2} \in \mathbb{R}^{n}$. Also, we can find a positive constant $C_{\mathscr{H}}$ such that $\|\mathscr{H}(t, w)\| \leq C_{\mathscr{H}}$, for all $t \in \mathscr{J}_{1}$ and $w \in \mathbb{R}^{n}$.

(H3) The function $r: \mathscr{C}\left(\mathscr{J}, \mathbb{R}^{n}\right) \rightarrow \mathbb{R}^{n}$ is continuous in a way that

$$
\|r(w)\|_{\mathbb{R}^{n}} \leq c\|w\|_{\mathscr{C}}^{\eta}+c_{0},
$$

with $0<\eta \leq 1$

(H4) The function $r: \mathscr{C}\left(\mathscr{J}, \mathbb{R}^{n}\right) \rightarrow \mathbb{R}^{n}$ is continuous and we can find a positive constant $c_{1}$ in a way that

$$
\left\|r\left(w_{1}\right)-r\left(w_{2}\right)\right\|_{\mathbb{R}^{n}} \leq c_{1}\left\|w_{1}-w_{2}\right\|_{\mathscr{C}}
$$

(H5) The functions $\xi_{q}: I_{q} \times \mathbb{R}^{n} \rightarrow \mathbb{R}^{n}, I_{q}=\left[t_{q}, s_{q}\right], q=1,2, \ldots, p$ are continuous such that

$$
\left\|\xi_{q}(t, x)\right\|_{\mathbb{R}^{n}} \leq \gamma_{q}\|w\|_{\mathbb{R}^{n}}^{\beta_{q}}+\delta_{q}, t \in I_{q}, w \in \mathbb{R}^{n}
$$

with $0<\beta_{q} \leq 1, q=1,2, \ldots, p$.

(H6) The functions $\xi_{q}: I_{q} \times \mathbb{R}^{n} \rightarrow \mathbb{R}^{n}$ are continuous and there exist constants $\mathscr{L}_{\xi_{q}}>0, q=1,2, \ldots, p$, in ways that $\left\|\xi_{q}\left(t, w_{1}\right)-\xi_{q}\left(s, w_{2}\right)\right\|_{\mathbb{R}^{n}} \leq \mathscr{L}_{\xi_{q}}\left(|t-s|+\left\|w_{1}-w_{2}\right\|_{\mathbb{R}^{n}}\right), \forall t, s, \in I_{q}, w_{1}, w_{2} \in \mathbb{R}^{n}$. 
CONTROLLABILITY OF NONLOCAL NON-AUTONOMOUS NEUTRAL DIFFERENTIAL SYSTEMS INCLUDING NON-INSTANTANEOUS IMPULSIVE EFFECTS IN $\mathbb{R}^{n}$

Now, we are in a position to define the mild solution for the system (1.1)$(1.3)$.

Definition 2.2. A function $w(t) \in \mathbb{R}^{n}$ is called a mild solution of the system (1.1)-(1.3) if $w(0)=w_{0}+r(w), w(t)=\xi_{q}\left(t, w\left(t_{q}^{-}\right)\right)$for all $t \in\left(t_{q}, s_{q}\right], q=$ $1,2, \ldots, p$ and $w(t)$ is the solution of the subsequent integral equations

$$
\begin{aligned}
w(t)= & U(t, 0)\left[w_{0}+r(w)-\mathscr{H}(0, w(0))\right]+\mathscr{H}(t, w(t))+\int_{0}^{t} U(t, s) B(s) u(s) d s \\
& +\int_{0}^{t} U(t, s) \mathscr{F}(s, w(s)) d s, \forall t \in\left[0, t_{1}\right], \\
w(t)= & U\left(t, s_{q}\right)\left[\xi_{q}\left(s_{q}, w\left(t_{q}^{-}\right)\right)-\mathscr{H}\left(s_{q}, w\left(t_{q}^{-}\right)\right)\right]+\mathscr{H}\left(t, w\left(t_{q}^{-}\right)\right) \\
& +\int_{s_{q}}^{t} U(t, s) B(s) u(s) d s+\int_{s_{q}}^{t} U(t, s) \mathscr{F}(s, w(s)) d s, \\
& \forall t \in\left(s_{q}, t_{q+1}\right], q=1,2, \ldots, p .
\end{aligned}
$$

\section{Controllability for Linear System}

Before we establish the controllability result of the problem (1.1)-(1.3), first, we discuss the subsequent problem having no impulsive conditions.

We accept that the nonlocal non-autonomous linear problem

$$
\begin{aligned}
& w^{\prime}(t)=A(t) w(t)+B(t) u(t), t \in \mathscr{J}, \\
& w(0)=w_{0}+r(w) \in \mathbb{R}^{n},
\end{aligned}
$$

admits unique solution given by

$$
w(t)=U(t, 0)\left[w_{0}+r(w)\right]+\int_{0}^{t} U(t, s) B(s) u(s) d s, \forall t \in \mathscr{J},
$$

where $U(t, s)=\Psi(t) \Psi^{-1}(s)$ and $\Psi(t)$ is the fundamental matrix of the system

$$
w^{\prime}(t)=A(t) w(t) .
$$

The matrix $\Psi(t)$ fulfills

$$
\begin{aligned}
& \Psi^{\prime}(t)=A(t) \Psi(t) \\
& \Psi(0)=I_{\mathbb{R}^{n}},
\end{aligned}
$$

where $I_{\mathbb{R}^{n}}$ is the $n \times n$ identity matrix. Consequently there exist constants $\mathscr{M}>0$ and $\omega>0$ in a way that

$$
\|U(t, s)\| \leq \mathscr{M} e^{\omega(t-s)}, \quad 0 \leq s \leq t \leq T .
$$


CONTROLLABILITY OF NONLOCAL NON-AUTONOMOUS NEUTRAL DIFFERENTIAL SYSTEMS INCLUDING NON-INSTANTANEOUS IMPULSIVE

Presently, we designate the map $\Upsilon: \mathscr{L}^{2}\left(\mathscr{J}, \mathbb{R}^{m}\right) \rightarrow \mathbb{R}^{n}$ is denoted by

$$
\Upsilon u=\int_{0}^{T} U(T, s) B(s) u(s) d s .
$$

The adjoint operator $\Upsilon^{*}: \mathbb{R}^{n} \rightarrow \mathscr{L}^{2}\left(\mathscr{J}, \mathbb{R}^{m}\right)$ of the operator $\Upsilon$ is denoted by

$$
\left(\Upsilon^{*} w\right)(s)=B^{*}(s) U^{*}(T, s) w
$$

In this situation, the controllability Grammian matrix $G_{0}^{T}: \mathbb{R}^{n} \rightarrow \mathbb{R}^{n}$ is given by

$$
G_{0}^{T} w=\int_{0}^{T} U(T, s) B(s) B^{*}(s) U^{*}(T, s) w(s) d s .
$$

Based on the above discussion, the problem (3.1)-(3.2) is controllable if and only if there exists a $\delta>0$ in a way that

$$
\left\langle G_{0}^{T} w, w\right\rangle \geq \delta\|w\|^{2}, \quad \forall w \in \mathbb{R}^{n},
$$

then $\left\|\left(G_{0}^{T}\right)^{-1}\right\| \leq \frac{1}{\delta}$.

Therefore, the operator $\bar{\Upsilon}: \mathbb{R}^{n} \rightarrow \mathscr{L}^{2}\left(\mathscr{J}, \mathbb{R}^{m}\right)$ defined by

$$
\bar{\Upsilon} w=B^{*}(\cdot) U^{*}(T, \cdot)\left(G_{0}^{T}\right)^{-1} w=\Upsilon^{*}\left(\Upsilon \Upsilon^{*}\right)^{-1} w
$$

is called the steering operator and is an inverse for the right of $\Upsilon$, include that $\Upsilon \bar{\Upsilon}=I$.

Presently, we characterize the steering control that move $w(0)$ to $w_{1}$ is given by

$$
u(t)=B^{*}(t) U^{*}(T, t)\left(G_{0}^{T}\right)^{-1}\left[w_{1}-U(T, 0)\left(w_{0}+r(w)\right)\right] .
$$

For additional concept on this theory, we suggest the reader to refer $[10$, $11,12]$.

\section{Main Results}

In this section, we present and prove the controllability results for the system (1.1)-(1.3) along with Rothe's fixed point theorem. Before, we prove the main theorem of this manuscript, initially, we need to prove the following two auxiliary results. 
CONTROLLABILITY OF NONLOCAL NON-AUTONOMOUS NEUTRAL

DIFFERENTIAL SYSTEMS INCLUDING NON-INSTANTANEOUS IMPULSIVE

EFFECTS IN $\mathbb{R}^{n}$

Lemma 4.1. If the conditions $(H 1)-(H 3)$ are hold, then the control function for the system (1.1)-(1.3) has $\|u(t)\| \leq Q_{1}, \forall t \in\left[0, t_{1}\right]$, where

$$
\begin{aligned}
Q_{1}= & \frac{\widetilde{\mathscr{M}}\|B\|}{\delta}\left[\left\|w_{t_{1}}\right\|+\mathscr{M}_{1}\left\{\left\|w_{0}\right\|+c\|w\|_{\mathscr{C}}^{\eta}+c_{0}-\mathscr{M}_{2}\right\}+\mathscr{M}_{2}\right. \\
& \left.+\mathscr{M}_{3}\left\{\gamma_{0}\|w\|_{\mathbb{R}^{n}}^{\beta_{0}}+\delta_{0}\right\}\right]
\end{aligned}
$$

where $\widetilde{\mathscr{M}}=\mathscr{M} e^{\omega\left(t_{1}-t\right)}, \mathscr{M}_{1}=\mathscr{M} e^{\omega t_{1}}, \mathscr{M}_{2}=C_{\mathscr{H}}$ and $\mathscr{M}_{3}=\frac{\mathscr{M}}{\omega}\left[e^{\omega t_{1}}-1\right]$.

Proof. For all $t \in\left[0, t_{1}\right]$, presently, we characterize the control function $u(t)$, we sustain

$$
u(t)=B^{*}(t) U^{*}\left(t_{1}, t\right)\left(G_{s_{0}}^{t_{1}}\right)^{-1} \widetilde{P}(w),
$$

where

$$
\begin{gathered}
\widetilde{P}(w)=w_{t_{1}}-U\left(t_{1}, 0\right)\left[w_{0}+r(w)-\mathscr{H}(0, w(0))\right]-\mathscr{H}\left(t_{1}, w\left(t_{1}\right)\right) \\
-\int_{0}^{t_{1}} U\left(t_{1}, s\right) \mathscr{F}(s, w(s)) d s .
\end{gathered}
$$

By the mild solution (2.1), we have final state at $t=t_{1}$

$$
\begin{aligned}
w\left(t_{1}\right)= & U\left(t_{1}, 0\right)\left[w_{0}+r(w)-\mathscr{H}(0, w(0))\right]+\mathscr{H}\left(t_{1}, w\left(t_{1}\right)\right) \\
& +\int_{0}^{t_{1}} U\left(t_{1}, s\right) \mathscr{F}(s, w(s)) d s+\int_{0}^{t_{1}} U\left(t_{1}, s\right) B(s) u(s) d s \\
= & U\left(t_{1}, 0\right)\left[w_{0}+r(w)-\mathscr{H}(0, w(0))\right]+\mathscr{H}\left(t_{1}, w\left(t_{1}\right)\right) \\
& +\int_{0}^{t_{1}} U\left(t_{1}, s\right) \mathscr{F}(s, w(s)) d s+\left(G_{s_{0}}^{t_{1}}\right)\left(G_{s_{0}}^{t_{1}}\right)^{-1}\left[w_{t_{1}}-U\left(t_{1}, 0\right)\left[w_{0}+r(w)\right.\right. \\
& \left.-\mathscr{H}(0, w(0))]-\mathscr{H}\left(t_{1}, w\left(t_{1}\right)\right)-\int_{0}^{t_{1}} U\left(t_{1}, s\right) \mathscr{F}(s, w(s)) d s\right] \\
= & w_{t_{1}} .
\end{aligned}
$$

Thus, the control (4.1) is appropriate for $t \in\left[0, t_{1}\right]$. 
CONTROLLABILITY OF NONLOCAL NON-AUTONOMOUS NEUTRAL

DIFFERENTIAL SYSTEMS INCLUDING NON-INSTANTANEOUS IMPULSIVE

EFFECTS IN $\mathbb{R}^{n}$

Presently, we sustain:

$$
\begin{aligned}
\|u(t)\| \leq & \frac{\|B\| \mathscr{M} e^{\omega\left(t_{1}-t\right)}}{\delta}\left[\left\|w_{t_{1}}\right\|+\mathscr{M} e^{\omega t_{1}}\left\{\left\|w_{0}\right\|+c\|w\|_{\mathscr{C}}^{\eta}+c_{0}-C_{\mathscr{H}}\right\}+C_{\mathscr{H}}\right. \\
& \left.+\int_{0}^{t_{1}} \mathscr{M} e^{\omega\left(t_{1}-s\right)}\left\{\gamma_{0}\|w\|_{\mathbb{R}^{n}}^{\beta_{0}}+\delta_{0}\right\} d s\right] \\
\leq & \frac{\widetilde{\mathscr{M}}\|B\|}{\delta}\left[\left\|w_{t_{1}}\right\|+\mathscr{M}_{1}\left\{\left\|w_{0}\right\|+c\|w\|_{\mathscr{C}}^{\eta}+c_{0}-\mathscr{M}_{2}\right\}+\mathscr{M}_{2}\right. \\
& \left.+\mathscr{M}_{3}\left\{\gamma_{0}\|w\|_{\mathbb{R}^{n}}^{\beta_{0}}+\delta_{0}\right\}\right] .
\end{aligned}
$$

This completes the proof.

Lemma 4.2. If the conditions $(H 1)-(H 3)$ and $(H 5)$ are hold, then the control function for the system (1.1)-(1.3) has $\|u(t)\| \leq Q_{q}, \forall t \in\left[s_{q}, t_{q+1}\right], q=$ $1,2, \ldots, p$, where

$$
\begin{aligned}
Q_{q}= & \frac{\overline{\mathscr{M}}\|B\|}{\delta_{q}}\left[\left\|w_{t_{q+1}}\right\|+\mathscr{M}_{4}\left\{\gamma_{q}\|w\|_{\mathbb{R}^{n}}^{\beta_{q}}+\delta_{q}\right\}+\left(\mathscr{M}_{4}+1\right) \mathscr{M}_{2}\right. \\
& \left.+\mathscr{M}_{5}\left\{\gamma_{0}\|w\|_{\mathbb{R}^{n}}^{\beta_{0}}+\delta_{0}\right\}\right],
\end{aligned}
$$

where $\overline{\mathscr{M}}=\mathscr{M} e^{\omega\left(t_{q+1}-t\right)}, \mathscr{M}_{4}=\mathscr{M} e^{\omega\left(t_{q+1}-s_{q}\right)}$ and $\mathscr{M}_{5}=\frac{\mathscr{M}}{\omega}\left[e^{\omega\left(t_{q+1}-s_{q}\right)}-\right.$ $1], q=1,2, \ldots, p$.

Proof. For all $t \in\left[s_{q}, t_{q+1}\right], q=1,2, \ldots, p$, presently, we characterize the control function $u(t)$, we sustain

$$
u(t)=B^{*}(t) U^{*}\left(t_{q+1}, t\right)\left(G_{s_{q}}^{t_{q+1}}\right)^{-1} \widetilde{P}_{1}(w),
$$

where

$$
\begin{aligned}
\widetilde{P}_{1}(w)= & w_{t_{q+1}}-U\left(t_{q+1}, s_{q}\right)\left[\xi_{q}\left(s_{q}, w\left(t_{q}^{-}\right)\right)-\mathscr{H}\left(s_{q}, w\left(t_{q}^{-}\right)\right)\right]-\mathscr{H}\left(t_{q+1}, w\left(t_{q}^{-}\right)\right) \\
& -\int_{s_{q}}^{t_{q+1}} U\left(t_{q+1}, s\right) \mathscr{F}(s, w(s)) d s,
\end{aligned}
$$

and

$$
G_{s_{q}}^{t_{q+1}} w=\int_{s_{q}}^{t_{q+1}} U\left(t_{q+1}, s\right) B(s) B^{*}(s) U^{*}\left(t_{q+1}, s\right) w(s) d s \text { and } \xi_{0}(0, \cdot)=w_{0}+r(w) .
$$


CONTROLLABILITY OF NONLOCAL NON-AUTONOMOUS NEUTRAL

We replacement the estimation of the $u(t)$ from (4.2) in (2.2), and supplant $t$ by $t_{q+1}, q=1,2, \ldots, p, w\left(t_{p+1}\right)=w_{t_{p+1}}=w_{1}$, we obtain

$$
\begin{aligned}
& w\left(t_{q+1}\right)=U\left(t_{q+1}, s_{q}\right)\left[\xi_{q}\left(s_{q}, w\left(t_{q}^{-}\right)\right)-\mathscr{H}\left(s_{q}, w\left(t_{q}^{-}\right)\right)\right]+\mathscr{H}\left(t_{q+1}, w\left(t_{q}^{-}\right)\right) \\
& +\int_{s_{q}}^{t_{q+1}} U\left(t_{q+1}, s\right) \mathscr{F}(s, w(s)) d s+\int_{s_{q}}^{t_{q+1}} U\left(t_{q+1}, s\right) B(s) u(s) d s \\
& =U\left(t_{q+1}, s_{q}\right)\left[\xi_{q}\left(s_{q}, w\left(t_{q}^{-}\right)\right)-\mathscr{H}\left(s_{q}, w\left(t_{q}^{-}\right)\right)\right]+\mathscr{H}\left(t_{q+1}, w\left(t_{q}^{-}\right)\right) \\
& +\int_{s_{q}}^{t_{q+1}} U\left(t_{q+1}, s\right) \mathscr{F}(s, w(s)) d s+\int_{s_{q}}^{t_{q+1}} U\left(t_{q+1}, s\right) B(s) B^{*}(s) \\
& (\times) U^{*}\left(t_{q+1}, s\right)\left(G_{s_{q}}^{t_{q+1}}\right)^{-1}\left[w_{t_{q+1}}-U\left(t_{q+1}, s_{q}\right)\left[\xi_{q}\left(s_{q}, w\left(t_{q}^{-}\right)\right)\right.\right. \\
& \left.-\mathscr{H}\left(s_{q}, w\left(t_{q}^{-}\right)\right)\right]-\mathscr{H}\left(t_{q+1}, w\left(t_{q}^{-}\right)\right) \\
& \left.-\int_{s_{q}}^{t_{q+1}} U\left(t_{q+1}, \eta\right) \mathscr{F}(\eta, w(\eta)) d \eta\right] d s \\
& =U\left(t_{q+1}, s_{q}\right)\left[\xi_{q}\left(s_{q}, w\left(t_{q}^{-}\right)\right)-\mathscr{H}\left(s_{q}, w\left(t_{q}^{-}\right)\right)\right]+\mathscr{H}\left(t_{q+1}, w\left(t_{q}^{-}\right)\right) \\
& +\int_{s_{q}}^{t_{q+1}} U\left(t_{q+1}, s\right) \mathscr{F}(s, w(s)) d s+\left(G_{s_{q}}^{t_{q+1}}\right)\left(G_{s_{q}}^{t_{q+1}}\right)^{-1}\left[w_{t_{q+1}}\right. \\
& -U\left(t_{q+1}, s_{q}\right)\left[\xi_{q}\left(s_{q}, w\left(t_{q}^{-}\right)\right)-\mathscr{H}\left(s_{q}, w\left(t_{q}^{-}\right)\right)\right]-\mathscr{H}\left(t_{q+1}, w\left(t_{q}^{-}\right)\right) \\
& \left.-\int_{s_{q}}^{t_{q+1}} U\left(t_{q+1}, \eta\right) \mathscr{F}(s, w(s)) d s\right] \\
& =w_{t_{q+1}} \text {. }
\end{aligned}
$$

Hence, the control function $u(t)$ given by (4.2) drive the state function $w(t)$ from starting state $w(0)$ to definite state $w_{1}$. Presently, we obtain

$$
\begin{aligned}
&\|u(t)\| \\
& \leq \frac{\|B\| \mathscr{M} e^{\omega\left(t_{q+1}-t\right)}}{\delta_{q}}\left[\left\|w_{t_{q+1}}\right\|+\left\|U\left(t_{q+1}, s_{q}\right)\right\|\left[\left\|\xi_{q}\left(s_{q}, w\left(t_{q}^{-}\right)\right)\right\|+\left\|\mathscr{H}\left(s_{q}, w\left(t_{q}^{-}\right)\right)\right\|\right]\right. \\
&\left.+\left\|\mathscr{H}\left(t_{q+1}, w\left(t_{q}^{-}\right)\right)\right\|+\int_{s_{q}}^{t_{q+1}}\left\|U\left(t_{q+1}, s\right)\right\| \mathscr{F}(s, w(s)) \| d s\right] \\
& \leq \frac{\bar{M}\|B\|}{\delta_{q}}\left[\left\|w_{t_{q+1}}\right\|+\mathscr{M}_{4}\left\{\gamma_{q}\|w\|_{\mathbb{R}^{n}}^{\beta_{q}}+\delta_{q}\right\}+\left(\mathscr{M}_{4}+1\right) \mathscr{M}_{2}+\mathscr{M}_{5}\left\{\gamma_{0}\|w\|_{\mathbb{R}^{n}}^{\beta_{0}}+\delta_{0}\right\}\right] \\
& \leq Q_{q}, \quad q=1,2, \ldots, p .
\end{aligned}
$$

The proof is now completed.

Now, we define the operator $\widetilde{\Upsilon}: \mathscr{P} \mathscr{C}\left(\mathscr{J}, \mathbb{R}^{n}\right) \times\left(\mathscr{J}, \mathbb{R}^{m}\right) \rightarrow \mathscr{P} \mathscr{C}\left(\mathscr{J}, \mathbb{R}^{n}\right) \times$ 
CONTROLLABILITY OF NONLOCAL NON-AUTONOMOUS NEUTRAL

$\left(\mathscr{J}, \mathbb{R}^{m}\right)$ by the subsequent form

$$
(z, v)=\left(\Upsilon_{1}(w, u), \Upsilon_{2}(w, u)\right)=\widetilde{\Upsilon}(w, u),
$$

where

$$
\begin{aligned}
z(t)= & \Upsilon_{1}(w, u)(t)=U(t, 0)\left[w_{0}+r(w)-\mathscr{H}(0, w(0))\right]+\mathscr{H}(t, w(t)) \\
& +\int_{0}^{t} U(t, s) \mathscr{F}(s, w(s)) d s+\int_{0}^{t} U(t, s) B(s)\left[\bar{\Upsilon} \lambda_{0}(w, u)\right] d s, \forall t \in\left[0, t_{1}\right] \\
z(t)= & \Upsilon_{1}(w, u)(t)=\xi_{q}\left(t, w\left(t_{q}^{-}\right)\right), \forall t \in\left(t_{q}, s_{q}\right], q=1,2 \ldots, p \\
z(t)= & \Upsilon_{1}(w, u)(t)=U\left(t, s_{q}\right)\left[\xi_{q}\left(s_{q}, w\left(t_{q}^{-}\right)\right)-\mathscr{H}\left(s_{q}, w\left(t_{q}^{-}\right)\right)\right]+\mathscr{H}\left(t, w\left(t_{q}^{-}\right)\right) \\
& +\int_{s_{q}}^{t} U(t, s) \mathscr{F}(s, w(s)) d s+\int_{s_{q}}^{t} U(t, s) B(s)\left[\bar{\Upsilon} \lambda_{q}(w, u)\right] d s \\
& \forall t \in\left(s_{q}, t_{q+1}\right], q=1,2, \ldots, p \\
v(t)= & \Upsilon_{2}(w, u)(t)=\left[\bar{\Upsilon} \lambda_{0}(w, u)\right]=B^{*}(t) U^{*}\left(t_{1}, t\right)\left(G_{s_{0}}^{t_{1}}\right)^{-1}\left[\lambda_{0}(w, u)\right], \forall t \in\left[0, t_{1}\right], \\
v(t)= & \Upsilon_{2}(w, u)(t)=\left[\bar{\Upsilon} \lambda_{q}(w, u)\right]=B^{*}(t) U^{*}\left(t_{q+1}, t\right)\left(G_{s_{q}}^{t_{q+1}}\right)^{-1}\left[\lambda_{q}(w, u)\right] \\
& \forall t \in\left[s_{q}, t_{q+1}\right], q=1,2, \ldots, p
\end{aligned}
$$

with

$$
\begin{aligned}
\lambda_{0}(w, u)= & w_{t_{1}}-U\left(t_{1}, 0\right)\left[w_{0}+r(w)-\mathscr{H}(0, w(0))\right]-\mathscr{H}\left(t_{1}, w\left(t_{1}\right)\right) \\
& -\int_{0}^{t_{1}} U\left(t_{1}, s\right) \mathscr{F}(s, w(s)) d s
\end{aligned}
$$

and

$$
\begin{aligned}
\lambda_{q}(w, u)= & w_{t_{q+1}}-U\left(t_{q+1}, s_{q}\right)\left[\xi_{q}\left(s_{q}, w\left(t_{q}^{-}\right)\right)-\mathscr{H}\left(s_{q}, w\left(t_{q}^{-}\right)\right)\right]-\mathscr{H}\left(t_{q+1}, w\left(t_{q}^{-}\right)\right) \\
& -\int_{s_{q}}^{t_{q+1}} U\left(t_{q+1}, s\right) \mathscr{F}(s, w(s)) d s, q=1,2, \ldots, p .
\end{aligned}
$$

Theorem 4.1. Suppose the conditions $(H 1)-(H 6)$ are fulfilled. The system (1.1)-(1.3) is controllable if and only if for all $w(0), w_{1} \in \mathbb{R}^{n}$, then operator $\widetilde{\Upsilon}(w, u)$ estimated in (4.3)-(4.9) has a fixed point. i.e.,

$$
\widetilde{\Upsilon}(w, u)=(w, u) .
$$

Now, we are in a position to present and prove a main theorem of this manuscript. 
CONTROLLABILITY OF NONLOCAL NON-AUTONOMOUS NEUTRAL DIFFERENTIAL SYSTEMS INCLUDING NON-INSTANTANEOUS IMPULSIVE

Theorem 4.2. Assume that the hypotheses $(H 1)-(H 6)$ are hold and the analogous linear system is controllable on $\mathscr{J}$. If $0<\beta_{q}<1, q=0,1,2, \ldots, p$ and $0<\eta<1$, then the problem (1.1)-(1.3) is controllable on $\mathscr{J}$. Furthermore, we can find a control $u \in\left(\mathscr{J}, \mathbb{R}^{m}\right)$ such that for a given $w(0), w_{1} \in \mathbb{R}^{n}$ the corresponding solution $w(\cdot)$ of system (1.1)-(1.3) fulfills

$$
\begin{aligned}
w_{t_{1}}= & U\left(t_{1}, 0\right)\left[w_{0}+r(w)-\mathscr{H}(0, w(0))\right]+\mathscr{H}\left(t_{1}, w\left(t_{1}\right)\right) \\
& +\int_{0}^{t_{1}} U\left(t_{1}, s\right) \mathscr{F}(s, w(s)) d s+\int_{0}^{t_{1}} U(t, s) B(s) u(s) d s, \\
w_{t_{q+1}}= & U\left(t_{q+1}, s_{q}\right)\left[\xi_{q}\left(s_{q}, w\left(t_{q}^{-}\right)\right)-\mathscr{H}\left(s_{q}, w\left(t_{q}^{-}\right)\right)\right]+\mathscr{H}\left(t_{q+1}, w\left(t_{q}^{-}\right)\right) \\
& +\int_{s_{q}}^{t_{q+1}} U\left(t_{q+1}, s\right) \mathscr{F}(s, w(s)) d s+\int_{s_{q}}^{t_{q+1}} U\left(t_{q+1}, s\right) B(s) u(s) d s, \\
& q=1,2, \ldots, p,
\end{aligned}
$$

and

$$
\begin{aligned}
& u(t)=B^{*}(t) U^{*}\left(t_{1}, t\right)\left(G_{s_{0}}^{t_{1}}\right)^{-1}\left[\lambda_{0}(w, u)\right], \quad \forall t \in\left[0, t_{1}\right] ; \\
& u(t)=B^{*}(t) U^{*}\left(t_{q+1}, t\right)\left(G_{s_{q}}^{t_{q}+1}\right)^{-1}\left[\lambda_{q}(w, u)\right], \quad \forall t \in\left[s_{q}, t_{q+1}\right], q=1,2, \ldots, p,
\end{aligned}
$$

where $\lambda_{0}(w, u)$ and $\lambda_{q}(w, u)$ are same as defined in (4.8) and (4.9) respectively.

Proof. For better readability, we break the argument of this theorem within 4 steps.

Step 1: The operator $\widetilde{\Upsilon}$ is continuous.

The continuity of $\Upsilon_{1}$ and $\Upsilon_{2}$ are directly pursue against the continuity of the functions [(H1)-(H6)] $\mathscr{H}(t, w), \mathscr{F}(t, w), r(w), \xi_{q}(t, w), q=1,2, \ldots, p$, and $\lambda_{q}, q=1,2, \ldots, p$ respectively (since $\bar{\Upsilon}$ is well defined earlier).

Step 2: Now, we intend to demonstrate that the operator $\widetilde{\Upsilon}$ is compact. Allow $D$ be a bounded subset of $\mathscr{P} \mathscr{C}\left(\mathscr{J}, \mathbb{R}^{n}\right) \times \mathscr{C}\left(\mathscr{J}, \mathbb{R}^{m}\right)$. It pursue that

$$
\begin{gathered}
\|\mathscr{F}(\cdot, w)\|_{0} \leq R_{1},\|\mathscr{G}(\cdot, w)\|_{0} \leq R_{2},\|r(w)\| \leq R_{3}, \\
\left\|\left(G_{s_{q}}^{t_{q+1}}\right)^{-1} \lambda_{q}(w, u)\right\| \leq R_{q+4}, q=0,1, \ldots, p, \\
\left\|\xi_{q}(\cdot, w)\right\|_{0} \leq R_{p+q+5}, q=1,2, \ldots, p, \forall(w, u) \in D .
\end{gathered}
$$

Therefore, $\widetilde{\Upsilon}$ is bounded.

Next, we prove that the operator $\widetilde{\Upsilon}$ is equi-continuous on $\mathscr{J}$. To prove this, let $0 \leq \tau_{2}<\tau_{3} \leq t_{1}$, we sustain

$$
\begin{aligned}
& \left\|\widetilde{\Upsilon}(w, u)\left(\tau_{3}\right)-\widetilde{\Upsilon}(w, u)\left(\tau_{2}\right)\right\|_{1} \\
& =\left\|\Upsilon_{1}(w, u)\left(\tau_{3}\right)-\Upsilon_{1}(w, u)\left(\tau_{2}\right)\right\|+\left\|\Upsilon_{2}(w, u)\left(\tau_{3}\right)-\Upsilon_{2}(w, u)\left(\tau_{2}\right)\right\| .
\end{aligned}
$$


CONTROLLABILITY OF NONLOCAL NON-AUTONOMOUS NEUTRAL

DIFFERENTIAL SYSTEMS INCLUDING NON-INSTANTANEOUS IMPULSIVE

EFFECTS IN $\mathbb{R}^{n}$

Now,

$$
\begin{aligned}
&\left\|\Upsilon_{1}(w, u)\left(\tau_{3}\right)-\Upsilon_{1}(w, u)\left(\tau_{2}\right)\right\| \\
& \leq\left\|U\left(\tau_{3}, 0\right)-U\left(\tau_{2}, 0\right)\right\|\left[\left\|w_{0}\right\|+\|r(w)\|+\mathscr{H}(0, w(0)) \|\right] \\
&+\left\|\mathscr{H}\left(\tau_{3}, w\left(\tau_{3}\right)\right)-\mathscr{H}\left(\tau_{2}, w\left(\tau_{2}\right)\right)\right\|+\int_{\tau_{2}}^{\tau_{3}}\left\|U\left(\tau_{3}, s\right)\right\|\|B(s)\|\|u(s)\| d s \\
&+\int_{0}^{\tau_{2}}\left\|U\left(\tau_{3}, s\right)-U\left(\tau_{2}, s\right)\right\|\|B(s)\|\|u(s)\| d s \\
&+\int_{\tau_{2}}^{\tau_{3}}\left\|U\left(\tau_{3}, s\right)\right\|\|\mathscr{F}(s, w(s))\| d s \\
&+\int_{0}^{\tau_{2}}\left\|U\left(\tau_{3}, s\right)-U\left(\tau_{2}, s\right)\right\|\|\mathscr{F}(s, w(s))\| d s
\end{aligned}
$$

and

$$
\begin{aligned}
& \left\|\Upsilon_{2}(w, u)\left(\tau_{3}\right)-\Upsilon_{2}(w, u)\left(\tau_{2}\right)\right\| \\
& \leq\left\|B^{*}\left(\tau_{3}\right) U^{*}\left(t_{1}, \tau_{3}\right)-B^{*}\left(\tau_{1}\right) U^{*}\left(t_{1}, \tau_{2}\right)\right\|\left[\left\|\left(G_{s_{0}}^{t_{1}}\right)^{-1}\right\|\left\|\lambda_{0}(w, u)\right\|\right]
\end{aligned}
$$

Let $s_{q}<\tau_{2}<\tau_{3} \leq t_{q+1}, q=1,2, \ldots, p$, we have

$$
\begin{aligned}
& \left\|\widetilde{\Upsilon}(w, u)\left(\tau_{3}\right)-\widetilde{\Upsilon}(w, u)\left(\tau_{2}\right)\right\| \\
& \leq\left\|\Upsilon_{1}(w, u)\left(\tau_{3}\right)-\Upsilon_{1}(w, u)\left(\tau_{2}\right)\right\|+\left\|\Upsilon_{2}(w, u)\left(\tau_{3}\right)-\Upsilon_{2}(w, u)\left(\tau_{2}\right)\right\| .
\end{aligned}
$$

Now,

$$
\begin{aligned}
&\left\|\Upsilon_{1}(w, u)\left(\tau_{3}\right)-\Upsilon_{1}(w, u)\left(\tau_{2}\right)\right\| \\
& \leq\left\|U\left(\tau_{3}, s_{q}\right)-U\left(\tau_{2}, s_{q}\right)\right\|\left[\left\|\xi_{q}\left(s_{q}, w\left(t_{q}^{-}\right)\right)\right\|+\left\|\mathscr{H}\left(s_{q}, w\left(t_{q}^{-}\right)\right)\right\|\right] \\
&+\left\|\mathscr{H}\left(\tau_{3}, w\left(t_{q}^{-}\right)\right)-\mathscr{H}\left(\tau_{2}, w\left(t_{q}^{-}\right)\right)\right\| \\
&+\int_{s_{q}}^{\tau_{2}}\left\|U\left(\tau_{3}, s\right)-U\left(\tau_{2}, s\right)\right\|\|B(s)\|\|u(s)\| d s+\int_{\tau_{2}}^{\tau_{3}}\left\|U\left(\tau_{3}, s\right)\right\|\|B(s)\|\|u(s)\| d s \\
&+\int_{s_{q}}^{\tau_{2}}\left\|U\left(\tau_{3}, s\right)-U\left(\tau_{2}, s\right)\right\|\|\mathscr{F}(s, w(s))\| d s+\int_{\tau_{2}}^{\tau_{3}}\left\|U\left(\tau_{3}, s\right)\right\|\|\mathscr{F}(s, w(s))\| d s
\end{aligned}
$$

and

$$
\begin{aligned}
& \left\|\Upsilon_{1}(w, u)\left(\tau_{3}\right)-\Upsilon_{1}(w, u)\left(\tau_{2}\right)\right\| \\
& \leq\left\|B^{*}\left(\tau_{3}\right) U^{*}\left(t_{q+1}, \tau_{3}\right)-B^{*}\left(\tau_{1}\right) U^{*}\left(t_{q+1}, \tau_{2}\right)\right\|\left[\left\|\left(G_{s_{q}}^{t_{q+1}}\right)^{-1}\right\|\left\|\lambda_{i}(w, u)\right\|\right] .
\end{aligned}
$$

Let $t_{q}<\tau_{2}<\tau_{3} \leq s_{q}, q=1,2, \ldots, p$, we have $\left\|\widetilde{\Upsilon}(w, u)\left(\tau_{3}\right)-\widetilde{\Upsilon}(w, u)\left(\tau_{2}\right)\right\| \leq\left\|\xi_{q}\left(\tau_{3}, w\left(t_{q}^{-}\right)\right)-\xi_{q}\left(\tau_{2}, w\left(t_{q}^{-}\right)\right)\right\| \leq \mathscr{L}_{\xi_{q}}\left|\tau_{3}-\tau_{2}\right|$. 
CONTROLLABILITY OF NONLOCAL NON-AUTONOMOUS NEUTRAL DIFFERENTIAL SYSTEMS INCLUDING NON-INSTANTANEOUS IMPULSIVE

Presume, if we select a sequence $\left\{\varphi_{j}\right\}$ against $\widetilde{\Upsilon}(D)$ which is equi-continuous and uniformly bounded on the interval $\left[0, t_{1}\right]$. Subsequently, by utilizing the Arzela theorem, there is a sub-sequence $\left\{\varphi_{j}^{1}\right\}$ of $\left\{\varphi_{j}\right\}$ which is uniformly convergent on $\left[0, t_{1}\right]$.

Presently, we consider sequence $\left\{\varphi_{j}^{1}\right\}$ on the interval $\left(t_{1}, t_{2}\right]$. Since the sequence $\left\{\varphi_{j}^{1}\right\}$ is equi-continuous and uniformly bounded on $\left(t_{1}, t_{2}\right]$. Thus, by making use of Arzela theorem, there is a sub-sequence $\left\{\varphi_{j}^{2}\right\}$ which is uniformly convergent on $\left[0, t_{2}\right]$.

Continuing this process for the intervals $\left(t_{2}, t_{3}\right],\left(t_{3}, t_{4}\right], \ldots\left(t_{p}, t_{p+1}\right]$, we observe that the sequence $\left\{\varphi_{j}^{p+1}\right\}$ converges uniformly on the interval $\mathscr{J}$. From the above discussion, we confirm that the operator $\widetilde{\Upsilon}$ is compact.

Step 3: In this case, we intend to demonstrate that the subsequent inequality

$$
\lim _{\|(w, u)\| \rightarrow \infty} \frac{\|\widetilde{\Upsilon}(w, u)\|}{\|(w, u)\|}=0
$$

where $\|(w, u)\|$ is the norm in the space $\mathscr{P} \mathscr{C}\left(\mathscr{J}, \mathbb{R}^{n}\right) \times \mathscr{C}\left(\mathscr{J}, \mathbb{R}^{m}\right)$.

To prove this, we need to furnish the following estimations:

$$
\begin{aligned}
\left\|\lambda_{0}(w, u)\right\| \leq & \left\|w_{t_{1}}\right\|+\left\|U\left(t_{1}, 0\right)\right\|\left[\left\|w_{0}\right\|+\|r(w)\|+\|\mathscr{H}(0, w(0))\|\right] \\
& +\left\|\mathscr{H}\left(t_{1}, w\left(t_{1}\right)\right)\right\|+\int_{0}^{t_{1}}\left\|U\left(t_{1}, s\right)\right\|\|\mathscr{F}(s, w(s))\| d s \\
\leq & \left\|w_{t_{1}}\right\|+\mathscr{M}_{1}\left\|w_{0}\right\|+\mathscr{M}_{2}\left(\mathscr{M}_{1}+1\right)+\mathscr{M}_{1}\left\{c\|w\|_{\mathscr{C}}^{\eta}+c_{0}\right\} \\
& +\mathscr{M}_{3}\left\{\gamma_{0}\|w\|_{\mathbb{R}^{n}}^{\beta_{0}}+\delta_{0}\right\},
\end{aligned}
$$

where $\mathscr{M}_{1}, \mathscr{M}_{2}$ and $\mathscr{M}_{3}$ are same as defined in Lemma 4.1.

$$
\begin{aligned}
\left\|\lambda_{q}(w, u)\right\| \leq & \left\|w_{t_{q+1}}\right\|+\left\|U\left(t_{q+1}, s_{q}\right)\right\|\left[\left\|\xi_{q}\left(s_{q}, w\left(t_{q}^{-}\right)\right)\right\|+\left\|\mathscr{H}\left(s_{q}, w\left(t_{q}^{-}\right)\right)\right\|\right] \\
& +\left\|\mathscr{H}\left(t_{q+1}, w\left(t_{q}^{-}\right)\right)\right\|+\int_{s_{q}}^{t_{q+1}}\left\|U\left(t_{q+1}, s\right)\right\|\|\mathscr{F}(s, w(s))\| d s \\
\leq & \left\|w_{t_{q+1}}\right\|+\mathscr{M}_{2}\left(\mathscr{M}_{4}+1\right)+\mathscr{M}_{4}\left\{\gamma_{q}\|w\|_{\mathbb{R}^{n}}^{\beta_{q}}+\delta_{q}\right\} \\
& +\mathscr{M}_{5}\left\{\gamma_{0}\|w\|_{\mathbb{R}^{n}}^{\beta_{0}}+\delta_{0}\right\}, q=1,2, \ldots, p,
\end{aligned}
$$

where $\mathscr{M}_{4}$ and $\mathscr{M}_{5}$ are same as defined in Lemma 4.2.

$$
\begin{aligned}
\| & \Upsilon_{2}(w, u) \| \\
\leq & \widetilde{\mathscr{M}}\|B\|\left\|\left(G_{s_{0}}^{t_{1}}\right)^{-1}\right\|\left\{\left\|w_{t_{1}}\right\|+\mathscr{M}_{1}\left\|w_{0}\right\|\right\}+\widetilde{\mathscr{M}}\|B\|\left\|\left(G_{s_{0}}^{t_{1}}\right)^{-1}\right\|\left\{\mathscr{M}_{2}\left(1+\mathscr{M}_{1}\right)\right\} \\
& +\widetilde{\mathscr{M}} \mathscr{M}_{1}\|B\|\left\|\left(G_{s_{0}}^{t_{1}}\right)^{-1}\right\|\left\{c\|w\|_{\mathscr{C}}^{\eta}+c_{0}\right\}+\widetilde{\mathscr{M}} \mathscr{M}_{3}\|B\|\left\{\gamma_{0}\|w\|_{\mathbb{R}^{n}}^{\beta_{0}}+\delta_{0}\right\}, t \in\left[0, t_{1}\right],
\end{aligned}
$$


CONTROLLABILITY OF NONLOCAL NON-AUTONOMOUS NEUTRAL

DIFFERENTIAL SYSTEMS INCLUDING NON-INSTANTANEOUS IMPULSIVE

EFFECTS IN $\mathbb{R}^{n}$

where $\widetilde{\mathscr{M}}$ is same as defined in Lemma 4.1 .

$$
\begin{aligned}
& \left\|\Upsilon_{2}(w, u)\right\| \\
& \leq \overline{\mathscr{M}}\|B\|\left\|\left(G_{s_{q}}^{t_{q+1}}\right)^{-1}\right\|\left\{\left\|w_{t_{q+1}}\right\|+\left(\mathscr{M}_{4}+1\right) \mathscr{M}_{2}\right\}+\overline{\mathscr{M}} \mathscr{M}_{5}\|B\|\left\|\left(G_{s_{q}}^{t_{q+1}}\right)^{-1}\right\| \\
& \quad \cdot\left\{\gamma_{q}\|w\|_{\mathbb{R}^{n}}^{\beta_{q}}+\delta_{q}\right\}+\overline{\mathscr{M}} \mathscr{M}_{5}\|B\|\left\|\left(G_{s_{q}}^{t_{q+1}}\right)^{-1}\right\|\left\{\gamma_{0}\|w\|_{\mathbb{R}^{n}}^{\beta_{0}}+\delta_{0}\right\} \\
& \quad t \in\left(s_{q}, t_{q+1}\right], q=1,2, \ldots, p
\end{aligned}
$$

where $\overline{\mathscr{M}}$ is same as defined in Lemma 4.2 .

$$
\begin{aligned}
& \left\|\Upsilon_{1}(w, u)\right\| \\
& \leq\left[\widetilde{\mathscr{M}_{1}}+\mathscr{M}_{1} \widetilde{\mathscr{M}_{3}}\|B\|^{2}\left\|\left(G_{s_{0}}^{t_{1}}\right)^{-1}\right\|\right]\left\{c\|w\|_{\mathscr{C}}^{\eta}+c_{0}\right\}+\widetilde{\mathscr{M}_{3}}\left[1+\mathscr{M}_{3} \widetilde{\mathscr{M}}\|B\|^{2}\left\|\left(G_{s_{0}}^{t_{1}}\right)^{-1}\right\|\right] \\
& \quad(\times)\left\{\gamma_{0}\|w\|_{\mathbb{R}^{n}}^{\beta_{0}}+\delta_{0}\right\}+\Lambda_{1}, t \in\left[0, t_{1}\right],
\end{aligned}
$$

where

$$
\begin{aligned}
& \Lambda_{1}=\widetilde{\mathscr{M}}_{1}\left\|w_{0}\right\|+\mathscr{M}_{2}\left(\widetilde{\mathscr{M}_{1}}+1\right)+\widetilde{\mathscr{M}_{3}} \widetilde{\mathscr{M}}\|B\|^{2}\left\|\left(G_{s_{0}}^{t_{1}}\right)^{-1}\right\|\left[\left\|w_{t_{1}}\right\|+\mathscr{M}_{1}\left\|w_{0}\right\|\right. \\
& \left.+\left\{\mathscr{M}_{2}\left(1+\mathscr{M}_{1}\right)\right\}\right] . \\
& \left\|\Upsilon_{1}(w, u)\right\| \leq\left[\overline{\mathscr{M}}_{1}+\overline{\mathscr{M}}_{3} \overline{\mathscr{M}}^{\left.\mathscr{M}_{4}\|B\|^{2}\left\|\left(G_{s_{q}}^{t_{q+1}}\right)^{-1}\right\|\right]\left\{\gamma_{q}\|w\|_{\mathbb{R}^{n}}^{\beta_{q}}+\delta_{q}\right\}}\right. \\
& \quad+\overline{\mathscr{M}}_{3}\left[1+\overline{\mathscr{M}}_{5}\|B\|^{2}\left\|\left(G_{s_{q}}^{t_{q+1}}\right)^{-1}\right\|\right]\left\{\gamma_{0}\|w\|_{\mathbb{R}^{n}}^{\beta_{0}}+\delta_{0}\right\}+\Lambda_{2}, \\
& t \in\left(s_{q}, t_{q+1}\right), q=1,2, \ldots, p,
\end{aligned}
$$

where

$$
\begin{gathered}
\Lambda_{2}=\mathscr{M}_{2}\left(1+\overline{\mathscr{M}}_{1}\right)+\overline{\mathscr{M}} \mathscr{M}_{3}\left\|\left(G_{s_{q}}^{t_{q+1}}\right)^{-1}\right\|\left\{\left\|w_{q+1}\right\|+\mathscr{M}_{2}\left(1+\mathscr{M}_{4}\right)\right\} \\
\quad t \in\left(s_{q}, t_{q+1}\right], q=1,2, \ldots, p . \\
\left\|\Upsilon_{1}(w, u)\right\| \leq\left\{\gamma_{q}\|w\|_{\mathbb{R}^{n}}^{\beta_{q}}+\delta_{q}\right\}, \forall t \in\left(t_{q}, s_{q}\right], q=1,2, \ldots, p .
\end{gathered}
$$

Therefore, we sustain

$$
\begin{aligned}
\| & \widetilde{\Upsilon}(w, u) \| \\
\leq & \left\|\Upsilon_{1}(w, u)\right\|+\left\|\Upsilon_{2}(w, u)\right\| \\
\leq & {\left[\widetilde{\mathscr{M}_{1}}+\mathscr{M}_{1} \widetilde{\mathscr{M}}\|B\|\left\|\left(G_{s_{0}}^{t_{1}}\right)^{-1}\right\|\left(1+\widetilde{\mathscr{M}_{3}}\|B\|\right)\right]\left\{c\|w\|_{\mathscr{C}}^{\eta}+c_{0}\right\}+\left[\widetilde{\mathscr{M}_{3}}\right.} \\
& \left.+\widetilde{\mathscr{M}} \mathscr{M}_{3}\|B\|\left(1+\widetilde{\mathscr{M}_{3}}\|B\|\right)\left\|\left(G_{s_{0}}^{t_{1}}\right)^{-1}\right\|\right]\left\{\gamma_{0}\|w\|_{\mathbb{R}^{n}}^{\beta_{0}}+\delta_{0}\right\}+\bar{\Lambda}_{1}, t \in\left[0, t_{1}\right] \\
\|\widetilde{\Upsilon}(w, u)\| &
\end{aligned}
$$


CONTROLLABILITY OF NONLOCAL NON-AUTONOMOUS NEUTRAL

DIFFERENTIAL SYSTEMS INCLUDING NON-INSTANTANEOUS IMPULSIVE

EFFECTS IN $\mathbb{R}^{n}$

$$
\begin{aligned}
& \leq\left[\overline{\mathscr{M}}_{1}+\|B\|\left\|\left(G_{s_{q}}^{t_{q+1}}\right)^{-1}\right\|\left(\overline{\mathscr{M}} \mathscr{M}_{5}+\mathscr{M} \mathscr{M}_{4} \overline{\mathscr{M}}_{3}\|B\|\right)\right]\left\{\gamma_{q}\|w\|_{\mathbb{R}^{n}}^{\beta_{q}}+\delta_{q}\right\} \\
& \quad+\left[\overline{\mathscr{M}}_{3}+\overline{\mathscr{M}} \mathscr{M}_{5}\|B\|\left\|\left(G_{s_{q}}^{t_{q+1}}\right)^{-1}\right\|\left(1+\overline{\mathscr{M}}_{3}\|B\|\right)\right]\left\{\gamma_{0}\|w\|_{\mathbb{R}^{n}}^{\beta_{0}}+\delta_{0}\right\}+\bar{\Lambda}_{2}, \\
& \quad t \in\left(s_{q}, t_{q+1}\right], q=1,2, \ldots, p, \\
& \|\widetilde{\Upsilon}(w, u)\| \leq\left\{\gamma_{q}\|w\|_{\mathbb{R}^{n}}^{\beta_{q}}+\delta_{q}\right\}, t \in\left(t_{q}, s_{q}\right], q=1,2, \ldots, p,
\end{aligned}
$$

where

$$
\begin{gathered}
\bar{\Lambda}_{1}=\widetilde{\mathscr{M}_{1}}\|B\|\left\|\left(G_{s_{0}}^{t_{1}}\right)^{-1}\right\|\left(1+\widetilde{\mathscr{M}_{3}}\|B\|\right)\left\|w_{t_{1}}\right\|+\widetilde{\mathscr{M}_{1}} \widetilde{\mathscr{M}}\|B\| \|\left(G_{s_{0}}^{t_{1}}\right)^{-1} \\
(\times)\left\|\left(1+\widetilde{\mathscr{M}_{3}}\|B\|\right)\right\| w_{0}\left\|+\widetilde{\mathscr{M}_{1}}\right\| w_{0}\left\|+\mathscr{\mathscr { M }}_{2}\left(1+\widetilde{\mathscr{M}_{1}}\right)+\widetilde{\mathscr{M}}\right\| B\|\|\left(G_{s_{0}}^{t_{1}}\right)^{-1} \\
(\times) \|\left(1+\widetilde{\mathscr{M}_{3}}\|B\|\right)\left[\mathscr{M}_{2}\left(1+\mathscr{M}_{1}\right)\right]
\end{gathered}
$$

and

$$
\begin{gathered}
\bar{\Lambda}_{2}=\overline{\mathscr{M}}\|B\|\left\|\left(G_{s_{q}}^{t_{q+1}}\right)^{-1}\right\|\left\{\left\|w_{q+1}\right\|\right. \\
\left.+\mathscr{M}_{2}\left(1+M_{4}\right)\right\}\left[1+\overline{\mathscr{M}}_{3}\|B\|\right]+\mathscr{\mathscr { M }}_{2}\left(1+\widetilde{\mathscr{M}}_{1}\right), q=1,2, \ldots, p .
\end{gathered}
$$

Hence,

$$
\begin{aligned}
& \frac{\|\widetilde{\Upsilon}(w, u)\|}{\|(w, u)\|} \leq \frac{\bar{\Lambda}_{1}}{\|w\|+\|u\|}+\left[\widetilde{\mathscr{M}_{1}}+\mathscr{M}_{1} \widetilde{\mathscr{M}}\|B\|\left\|\left(G_{s_{0}}^{t_{1}}\right)^{-1}\right\|\left(1+\widetilde{\mathscr{M}_{3}}\|B\|\right)\right] \\
& (\times)\left\{c\|w\|_{\mathscr{C}}^{\eta-1}+\frac{c_{0}}{\|w\|+\|u\|}\right\}+\left[\widetilde{\mathscr{M}_{3}}+\widetilde{\mathscr{M}} \mathscr{M}_{3}\|B\|\left(1+\widetilde{\mathscr{M}_{3}}\|B\|\right)\right. \\
& \left.(\times)\left\|\left(G_{s_{0}}^{t_{1}}\right)^{-1}\right\|\right]\left\{\gamma_{0}\|w\|_{\mathbb{R}^{n}}^{\beta_{0}-1}+\frac{\delta_{0}}{\|w\|+\|u\|}\right\} ;
\end{aligned}
$$

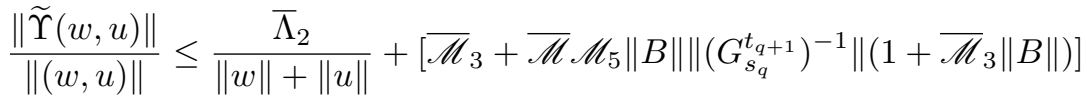

$$
\begin{aligned}
& (\times)\left\{\gamma_{0}\|w\|_{\mathbb{R}^{n}}^{\beta_{0}-1}+\frac{\delta_{0}}{\|w\|+\|u\|}\right\}+\left[\overline{\mathscr{M}}_{1}+\|B\|\left\|\left(G_{s_{q}}^{t_{q+1}}\right)^{-1}\right\|\right. \\
& \left.(\times)\left(\overline{\mathscr{M}}_{\mathscr{M}_{5}}+\mathscr{M} \mathscr{M}_{4} \overline{\mathscr{M}}_{3}\|B\|\right)\right]\left\{\gamma_{q}\|w\|_{\mathbb{R}^{n}}^{\beta_{q}-1}+\frac{\delta_{q}}{\|w\|+\|u\|}\right\} ; \\
& \frac{\|\widetilde{\Upsilon}(w, u)\|}{\|(w, u)\|} \leq\left\{\gamma_{q}\|w\|_{\mathbb{R}^{n}}^{\beta_{q}-1}+\frac{\delta_{q}}{\|w\|+\|u\|}\right\} .
\end{aligned}
$$

Hence, we have

$$
\lim _{\|(w, u)\| \rightarrow \infty} \frac{\|\widetilde{\Upsilon}(w, u)\|}{\|(w, u)\|}=0
$$


CONTROLLABILITY OF NONLOCAL NON-AUTONOMOUS NEUTRAL DIFFERENTIAL SYSTEMS INCLUDING NON-INSTANTANEOUS IMPULSIVE

Step 4: Finally, we will demonstrate that the operator $\widetilde{\Upsilon}$ has a fixed point. that

Indeed, for a fixed $0<\alpha<1$, there exists $r>0$ large enough in a way

$$
\|\widetilde{\Upsilon}(w, u)\| \leq \alpha\|(w, u)\| \quad \text { and } \quad\|(w, u)\|=r .
$$

Let $B(0, r)$ is a ball of radius $r>0$ and center zero, we have that $\widetilde{\Upsilon}(\partial B(0, r)) \subset B(0, r)$. In view of the maps the sphere $\partial B(0, r)$ into the interior of the ball $B(0, r)$ and $\widetilde{\Upsilon}$ is compact.

Presently, we are able to utilize the Rothe's fixed point theorem to guarantee the existence of a fixed point $(w, u) \in B(0, r) \subset \mathscr{P} \mathscr{C}\left(\mathscr{J}, \mathbb{R}^{n}\right) \times C\left(\mathscr{J}, \mathbb{R}^{m}\right)$ such that

$$
u=\bar{\Upsilon} \lambda_{0}(w, u)=B^{*}(\cdot) U^{*}\left(t_{1}, \cdot\right)\left(G_{s_{0}}^{t_{1}}\right)^{-1} \lambda_{0}(w, u), t \in\left[0, t_{1}\right] .
$$

Then

$$
\begin{aligned}
\Upsilon u= & \lambda_{0}(w, u) \\
= & w_{t_{1}}-U\left(t_{1}, 0\right)\left[w_{0}+r(w)-\mathscr{H}(0, w(0))\right]-\mathscr{H}\left(t_{1}, w\left(t_{1}\right)\right) \\
& -\int_{0}^{t_{1}} U\left(t_{1}, s\right) \mathscr{F}(s, w(s)) d s .
\end{aligned}
$$

Therefore

$$
\begin{aligned}
& \int_{0}^{t_{1}} U\left(t_{1}, s\right) B(s) u(s) d s \\
& =w_{t_{1}}-U\left(t_{1}, 0\right)\left[w_{0}+r(w)-\mathscr{H}(0, w(0))\right]-\mathscr{H}\left(t_{1}, w\left(t_{1}\right)\right) \\
& \quad-\int_{0}^{t_{1}} U\left(t_{1}, s\right) \mathscr{F}(s, w(s)) d s .
\end{aligned}
$$

Similarly for $t \in\left(s_{q}, t_{q+1}\right], q=1,2, \ldots, p$, we have

$$
\begin{gathered}
u=\bar{\Upsilon} \lambda_{q}(w, u)=B^{*}(\cdot) U^{*}\left(t_{q+1}, \cdot\right)\left(G_{s_{q}}^{t_{q+1}}\right)^{-1} \lambda_{q}(w, u), \\
t \in\left(s_{q}, t_{q+1}\right], q=1,2, \ldots, p .
\end{gathered}
$$

Then

$$
\begin{aligned}
\Upsilon u= & \lambda_{q}(w, u) \\
= & w_{t_{q+1}}-U\left(t_{q+1}, s_{q}\right)\left[\xi_{q}\left(s_{q}, w\left(t_{q}^{-}\right)\right)-\mathscr{H}\left(s_{q}, w\left(t_{q}^{-}\right)\right)\right]-\mathscr{H}\left(t_{q+1}, w\left(t_{q}^{-}\right)\right) \\
& -\int_{s_{q}}^{t_{q+1}} U\left(t_{q+1}, s\right) \mathscr{F}(s, w(s)) d s .
\end{aligned}
$$


CONTROLLABILITY OF NONLOCAL NON-AUTONOMOUS NEUTRAL DIFFERENTIAL SYSTEMS INCLUDING NON-INSTANTANEOUS IMPULSIVE

Therefore

$$
\begin{aligned}
& \int_{s_{q}}^{t_{q+1}} U\left(t_{q+1}, s\right) B(s) u(s) d s \\
& =w_{t_{q+1}}-U\left(t_{q+1}, s_{q}\right)\left[\xi_{q}\left(s_{q}, w\left(t_{q}^{-}\right)\right)-\mathscr{H}\left(s_{q}, w\left(t_{q}^{-}\right)\right)\right]-\mathscr{H}\left(t_{q+1}, w\left(t_{q}^{-}\right)\right) \\
& \quad-\int_{s_{q}}^{t_{q+1}} U\left(t_{q+1}, s\right) \mathscr{F}(s, w(s)) d s .
\end{aligned}
$$

\section{Acknowledgment}

The second author thanks to Dr. E. Kamalanaban, Principal, Vel Tech High Tech Dr. Rangarajan Dr. Sakunthala Engineering College, Avadi, Chennai-600062, Tamil Nadu, India, for his constant encouragement and support for this research work.

\section{References}

[1] R. Agarwal, S. Hristova and D.O'Regan, Non-Instantaneous Impulses in Differential Equations, Springer, 2017.

[2] S. Anju and Sanjay K. Srivastava, Lyapunov approach for stability of integro-differential equations with non-instantaneous impulse effect, Malaya Journal of Matematik, 4(1)(2016), 119-125.

[3] F.S. Acharya, Controllability of second order semilinear impulsive partial neutral functional differential equations with infinite delay, International Journal of Mathematics and Mathematical Sciences, 3(1)(2013), 207-218.

[4] L. Bai and J.J. Nieto, Variational approach to differential equations with not instantaneous impulses, Applied Mathematics Letters, 73(2017), 4448.

[5] L. Byszewski, Theorem about the existence and uniqueness of solutions of a semilinear evolution nonlocal Cauchy problem, Journal of Mathematical Analysis and Applications, 162(1991), 494-505.

[6] P. Chen, X. Zhang and Y. Li, Existence of mild solutions to partial differential equations with non-instantaneous impulses, Electronic Journal of Differential Equations, 241(2016), 1-11. 
CONTROLLABILITY OF NONLOCAL NON-AUTONOMOUS NEUTRAL DIFFERENTIAL SYSTEMS INCLUDING NON-INSTANTANEOUS IMPULSIVE

[7] K. Deng, Exponential decay of solutions of semilinear parabolic equations with nonlocal initial conditions, Journal of Mathematical Analysis and Applications, 179(1993), 630-637.

[8] E. Hernandez and D. ORegan, On a new class of abstract impulsive differential equations, Proceedings of the American Mathematical Society, 141(2013), 1641-1649.

[9] P. Kumar, D.N. Pandey, D. Bahuguna, On a new class of abstract impulsive functional differential equations of fractional order, Journal of Nonlinear Sciences and Applications, 7(2014), 102-114.

[10] H. Leiva, Rothe's fixed point theorem and controllability of semilinear non-autonomous systems, Systems \& Control Letters, 67(2014), 14-18.

[11] H. Leiva, Controllability of semilinear impulsive non-autonomous systems, International Journal of Control , 88(3)(2015), 585-592.

[12] M. Malik, R. Dhayal, S. Abbas and A. Kumar, Controllability of nonautonomous nonlinear differential system with non-instantaneous impulses, RASCAM, 113(1)(2019), 103-118.

[13] M. Pierri, D. ORegan and V. Rolnik, Existence of solutions for semi-linear abstract differential equations with non instantaneous impulses, Applied Mathematics and Computation, 219(2013), 6743-6749.

[14] R. Poongodi, V. T. Suveetha and S. Dhanalakshmi, Existence of mild solutions to partial neutral differential equations with non-instantaneous impulses, Malaya Journal of Matematik, 7(1)(2019), 27-33.

[15] S. Selvarasu, P. Kalamani and M. Mallika Arjunan, Approximate controllability of nonlocal impulsive fractional neutral stochastic integrodifferential equations with state-dependent delay in Hilbert spaces, Malaya Journal of Matematik, 4(4)(2016), 571-598.

[16] J. Wang and X. Li, Periodic BVP for integer/fractional order nonlinear differential equations with non-instantaneous impulses, Journal of Applied Mathematics and Computing, 46(2014), 321-334.

[17] J. Wang and M. Feckan, A general class of impulsive evolution equations, Topological Methods in Nonlinear Analysis, 46(2015), 915-933. 
CONTROLLABILITY OF NONLOCAL NON-AUTONOMOUS NEUTRAL

DIFFERENTIAL SYSTEMS INCLUDING NON-INSTANTANEOUS IMPULSIVE

EFFECTS IN $\mathbb{R}^{n}$

Velusamy Kavitha

Department of Mathematics,

School of Sciences, Arts, Media \& Management,

Karunya Institute of Technology and Sciences,

Karunya Nagar, Coimbatore-641114,

Tamil Nadu, India.

Email: kavi_velubagyam@yahoo.co.in

Mani Mallika Arjunan

Department of Mathematics,

Vel Tech High Tech Dr. Rangarajan Dr. Sakunthala Engineering College,

Avadi, Chennai-600062,

Tamil Nadu, India.

Email: arjunphd07@yahoo.co.in

Dumitru Baleanu

Department of Mathematics and Computer Sciences,

Faculty of Arts and Sciences, Cankaya University, 06530 Ankara, Turkey and

Institute of Space Sciences,

Magurele-Bucharest, Romania.

Email: dumitru@cankaya.edu.tr 